P152 Food Insecurity, Food Skills, and Household Food Availability Among Emerging Adults

Nicole Larson, PhD, MPH, RDN, larsonn@umn.edu, University of Minnesota, School of Public Health, $1300 \mathrm{~S}$ Second Street, Suite 300, Minneapolis, MN 55454; Melissa Laska, PhD, RD; Dianne Neumark-Sztainer, PhD, $M P H, R D$

Background (Background, Rationale, Prior Research, and/or Theory): Research regarding the involvement of emerging adults in household food activities is lacking to inform strategies for reducing food insecurity.

Objective: This study examines the prevalence of food insecurity among a population-based sample of emerging adults (18-25 years) and associations with food skills and household food availability.

Study Design, Setting, Participants, Intervention: Household food situations are being assessed as part of EAT 2010-2018, a follow-up study of young people recruited at secondary schools in Minneapolis-St. Paul, Minnesota in 2009-2010. Follow-up survey collection will be completed in spring 2018. Preliminary data include the responses of 868 participants (59\% female, 72\% ethnic minority).

Outcome Measures and Analysis: Participants were asked to report confidence to plan meals, follow a recipe, prepare a meal from items on hand; use basic cooking techniques, and stay within a food budget; frequency of eating home-prepared meals; food shopping involvement; and at-home healthy food availability. Analysis identified participants as food insecure if they had ever been hungry or eaten less than they should in the past year because there was inadequate money for food.

Results: Preliminary analyses indicate the overall prevalence of food insecurity was 31\%. Approximately one quarter to more than half of these food-insecure participants reported lacking confidence to perform food preparation activities; however, confidence in food skills did not differ by food security status. Mean frequency of eating meals prepared at home and food shopping involvement also did not differ by food security status. In contrast, food-insecure participants were less likely to report they usually had fruit, vegetables, and whole-grain bread available at home. For example, fresh fruit was usually available to approximately $70 \%$ of those who were food secure versus only about half of food-insecure participants $(P<.001)$.

Conclusions and Implications: Deficits in food skills are common but not disproportionately high among emerging adults that experience food insecurity.

Funding: NIH.

\section{P153 How Does Social Support From Partners Affect Primigravid Food Choices and Behaviors?}

Emma Greenhill, BS, Bradley University; Rachel L. Vollmer, PhD, RDN, rvollmer@fsmail.bradley.edu, Bradley

University, 1501 W Bradley Avenue, Peoria, IL 61625
Background (Background, Rationale, Prior Research, and/or Theory): Although healthful food choices and behaviors during pregnancy are recommended, women often do not meet the recommendations due to pregnancy barriers. Social support could be an important determinant, although there is limited research on a father's emerging roles during this life-cycle phase.

Objective: Explore role of social support in pregnancy, differing perception of the father's role during pregnancy between pregnant mothers and fathers, and the influence social support from a partner may or may not have on a pregnant mom's food choices and behaviors.

Study Design, Setting, Participants, Intervention: First-time pregnant, cohabitating couples ( $\mathrm{n}=11$ pairs) across the United States who met the inclusion criteria completed one-on-one semi-structured phone interviews.

Outcome Measures and Analysis: Interviews were audio-recorded and transcribed. Content analysis was applied to the transcriptions by two investigators, independently, to identify themes among mothers and fathers. Investigators met to discuss themes and reach consensus.

Results: The themes developed from the phone interview transcripts aligned with the social cognitive theory. For example, personal and environmental factors that influenced the mother's diet and physical activity behaviors during pregnancy included barriers, outcome expectations, collective-efficacy, and self-regulation, and social support from the father. Additionally, a theme of reciprocal determinism was evident, in which dietary and physical activity behaviors of mothers and fathers influenced one another.

Conclusions and Implications: If nutrition professionals are attempting to motivate pregnant mothers to adhere to recommendations, it may be beneficial to include fathers in interventions. Furthermore, because fathers' behaviors are impacted by mothers' behaviors during pregnancy, efforts to target both parents would be ideal to improve health behaviors.

Funding: None.

\section{P154 Perceptions of Videos Designed to Portray Evidence-Based Feeding Practice Guidelines for Young Children}

Lesli Biediger-Friedman, PhD, MPH, RD, LB47@txstate.edu, Texas State University, 600 University Drive, San Marcos, TX 78666; Sylvia H. Crixell, PhD, RD; Danielle M. Kuhn, BS; Courtney Cary, BS

Background (Background, Rationale, Prior Research, and/or Theory): The search for effective strategies to educate parents regarding recommended feeding practices for their young children is of paramount importance. Videos are becoming a preferred modality for education. This study delves into what intended users (parents) actually want in video-based education. 